\title{
CoviD-19 Are telephone consultations the future of the NHS? The outcomes and experiences of an NHS urological service in moving to telemedicine
}

\author{
Authors: Agathoklis Efthymiadis, ${ }^{\mathrm{A}}$ Edward $\mathrm{JM}$ Hart, ${ }^{\mathrm{A}}$ Alexandra M Guy, ${ }^{\mathrm{A}}$ Rebecca Harry, ${ }^{\mathrm{B}}$ Tharani Mahesan, ${ }^{\mathrm{C}}$ \\ Wissam Abou Chedid, ${ }^{D}$ Santiago Uribe-Lewis ${ }^{E}$ and Matthew JA Perry ${ }^{F}$
}

\section{Objective}

To evaluate urology patient satisfaction with telephone consultations during the COVID-19 pandemic.

\section{Methods}

All patients who received a telephone appointment in a 1-month period were invited to complete a questionnaire. An adaption of the Telehealth Satisfaction Scale (TeSS) was used. Patient responses were compared based on type of clinic, age and gender.

\section{Results}

119 questionnaires were completed. The majority of responses to the adapted TeSS (Q1-7) were graded as 'Excellent', ranging from 79 (66\%) to 112 (94\%). 'Agree' responses ranged from $92(77 \%)$ to 117 (98\%) for questions (Q8-12), indicating high satisfaction. Patients consulted in post radical prostatectomy and PSA surveillance clinics gave a significantly greater number of 'Excellent' or 'Agree' responses. Older age was associated with a significantly greater number of 'Agree' responses to one item only. Responses were not affected by gender.

Conclusion

Our study demonstrates high overall satisfaction with the use of telephone consultations among urology patients. For some patients, telephone consultations are more suitable and may be utilised more frequently in the future. However, it is clear that in selected cases face-to-face consultations are required for safe, comprehensive clinical assessment.

KEYWORDS: Telemedicine, teleurology, SARS-CoV-2, COVID-19, coronavirus

DOI:10.7861/fhj.2020-0076

Authors: Asenior house officer Stokes Centre for Urology, Guildford, UK; Bdoctor's assistant, Stokes Centre for Urology, Guildford, UK; Curology registrar, Stokes Centre for Urology, Guildford, UK; Dsenior urology fellow, Stokes Centre for Urology, Guildford, UK; ${ }^{E}$ clinical scientist, Stokes Centre for Urology, Guildford, UK; ; ${ }^{F}$ consultant urological surgeon, Stokes Centre for Urology, Guildford, UK

\section{Introduction}

The COVID-19 pandemic has necessitated radical changes in healthcare worldwide, including urology. ${ }^{1}$ In a global effort to minimise disease transmission and reallocate resources appropriately, NHS organisations are undergoing profound reorganisation, while the public has been encouraged to 'Stay home, Protect the NHS, Save lives'.

As part of this, clinicians have had to integrate telemedicine into their daily practice. ${ }^{2}$ The World Health Organization (WHO) defines telemedicine as 'The delivery of health care services, where distance is a critical factor, by all health care professionals using information and communication technologies for the exchange of valid information for diagnosis, treatment and prevention of disease and injuries, research and evaluation, and for the continuing education of health care provider. ${ }^{3}$ The adoption of telemedicine has ensured that face-to-face contact is minimised; resources such as hospital beds and personal protective equipment (PPE) are allocated appropriately; and the surge of hospital admissions is controlled. ${ }^{4}$ Moreover, it enables patients with chronic conditions to have their follow up from the safety of their own home. ${ }^{5}$

The Stokes Centre for Urology at The Royal Surrey NHS Foundation Trust is a tertiary centre that offers treatment for benign urology, andrology, oncological urology, including brachytherapy and robotic surgery for bladder and prostate cancer, as well as care for acute urology conditions. In response to the COVID-19 pandemic, all face-to-face clinic appointments were replaced with telephone consultations, to minimise disease transmission.

The objective of this study was to establish the degree of patient satisfaction with telephone consultations. Through implementation of a patient satisfaction questionnaire, we aimed to gain greater insight into our patients' perspective, and gauge opinion on their use beyond the COVID-19 pandemic.

\section{Methods}

As per Trust policy, face-to-face clinic appointments were converted to telephone appointments immediately after the government enforced national lockdown on 23 March 2020. 
Prior to this, face-to-face appointments would predominantly consist of history and physical examination, but could also include assessment of vital signs, urinalysis, urodynamic studies and urgent investigations including blood tests and imaging if requested by the clinician.

Patients were informed of the change and given a time slot in which they could expect to receive a call from the clinician. Consultations consisted of audio call only, conducted via clinic room telephones. No video conferencing software was used. Patients were not offered face-to-face consultations in the first instance. If deemed necessary by the clinician following a telephone consult, patients were occasionally requested to attend the hospital for full clinical assessment including physical examination. Patients who had been referred for investigations under the 2-week rule kept their original appointments for, for example, flexible cystoscopy to investigate haematuria. In addition, those presenting with urological emergencies or with high-risk cancer requiring urgent surgery were provided with faceto-face consultations.

Patients were consulted for a variety of urological presentations, with clinic types broadly categorised as post radical prostatectomy, prostate specific antigen (PSA) surveillance, general, functional, and andrology follow up. The general urology clinic included patients presenting with lower urinary tract symptoms, haematuria and stones.

Patients were not individually assessed on their suitability for telephone clinic. Consequently, discussions on complex management decisions and breaking bad news were also conducted via telephone.

All patients who had been scheduled for telephone clinic between 23 March to 30 April 2020 were identified by the urology outpatient department. Patients were excluded if their telephone appointment was cancelled, either by them or by the hospital, as investigations were pending, or if they could not be reached. Patients under the age of 18 were excluded. All remaining patients were included in the study.

Patients were contacted via telephone during the first week of May 2020 and invited to complete a verbal questionnaire. The phone call was not pre-scheduled; however, patients were given the opportunity to be called back at a more convenient time. It was specified to all patients that results would be anonymised and verbal consent was obtained in order to use the data for our study. The questionnaire was read out by the caller. Patients that did not respond to the first call were called on at least two other occasions.

An adaptation of the Telehealth Satisfaction Scale (TeSS) was used, which consisted of seven questions, with responses graded on a Likert scale from 1-4 (1 = 'Poor', 2 = 'Fair', 3 = 'Good', $4=$ 'Excellent'). ${ }^{6}$ An additional five questions relating specifically to telephone consultations were included, graded on an 'Agree; Neither agree nor disagree; Disagree' scale. Respondents were also given the opportunity to provide any further comments and suggestions for improvement.

\section{Statistical analyses}

Questionnaire responses were collated on Microsoft Excel. Allstatistical analyses were performed within R statistical environment (ww.R-project.org). To assess an association between item outcome and type of clinic, age, or gender the item responses were categorised into 'Excellent' and 'Other' ('Good', 'Fair' or 'Poor') for the adapted TeSS (Q1-7) or into 'Agree' and 'Other' ('Neither agree nor disagree' or 'Disagree') for the additional questions 8 to 12 . Univariable logistic regression was used to
Table 1. Demographics of patients that completed the survey

$\begin{array}{lll}\text { Number of cases } & & 119 \\ \text { Median age [range] } & & 72[27-91] \\ \text { Gender, } \mathrm{n}(\%) & \text { Female } & 15(13) \\ & \text { Male } & 104(87) \\ & \text { Prostatectomy } & 36(30) \\ & \text { PSA surveillance } & 34(29) \\ \text { Clinic, n (\%) } & \text { General } & 24(20) \\ & \text { Functional } & 18(15) \\ & \text { Andrology } & 7(6)\end{array}$

assess the binary response to the explanatory variables (see supplementary material, S1). An effect of gender was assessed in the relevant subset of clinics (functional and general). Likelihood ratio tests explored model goodness-of-fit relative to the empty model.

\section{Results}

A total of 269 patients were identified, and 194 met the inclusion criteria. Of the 75 (28\%) patients that were excluded, 12 were due to patient non-attendance and 63 appointments were cancelled or rescheduled for a later time due to investigations being delayed by the COVID-19 pandemic.

Of the 194 eligible patients, 119 (61\%) completed the questionnaire, 62 (32\%) patients did not answer the telephone after being contacted at least twice, and $13(7 \%)$ patients answered the telephone but refused to complete the questionnaire.

The median age of patients was 72 years (range 27-91 years) (Table 1). Eight (7\%) patients were below 50 years of age, 41 (34\%) were $50-70$ years old, and 70 (59\%) were older than 70 years. $104(87 \%)$ patients were male and $15(13 \%)$ patients were female. 36 (30\%) patients had been consulted in the post radical prostatectomy follow-up clinic, $34(29 \%)$ in the PSA surveillance clinic, $24(20 \%)$ in the general urology clinic, $18(15 \%)$ in the functional urology clinic, and seven $(6 \%)$ in the andrology clinic.

The responses to the adapted TeSS and additional questions are summarised in Fig 1 and Tables 2 and 3.

The great majority of responses to the adapted TeSS (Q1-7) were graded as either 'Excellent' or 'Good' for each item (Fig 1, left panel, and Table 2). The number of 'Excellent' responses ranged from $79(66 \%)$ to $112(94 \%)$ per item, while the number of responses graded as 'Good' ranged from $6(5 \%)$ to $18(15 \%)$. The number of responses graded as 'Fair' ranged from 0 to 5 ( $4 \%)$ per item. The number of responses graded as 'Poor' ranged from 0 to $3(2 \%)$. A higher proportion of 'No answer' responses were observed in questions three (explanation of diagnosis) and four (explanation of treatment), with 19 (16\%) and 26 (22\%) responses respectively.

Similarly, most patients responded 'Agree' to the additional questions (Q8-12), indicating a high degree of satisfaction 

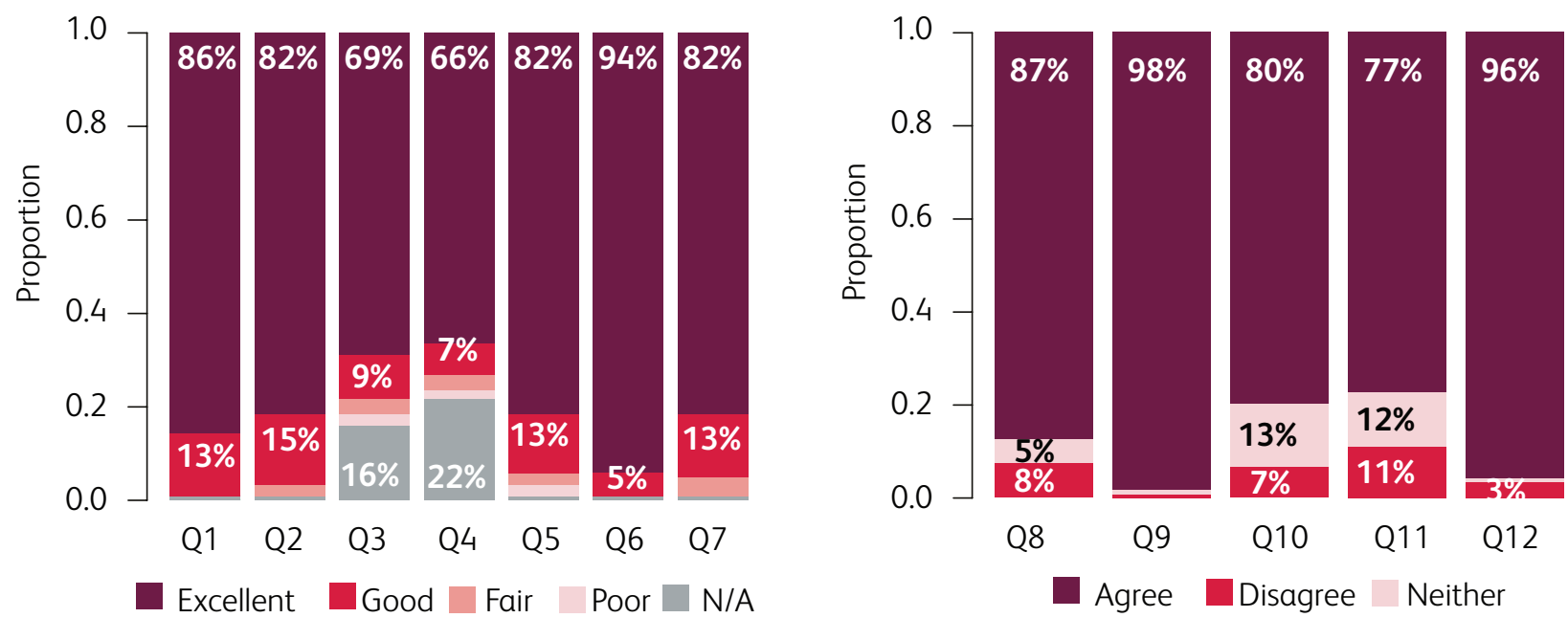

Fig 1. Overall responses to the Adapted Telehealth Satisfaction Scale (TeSS) and additional questions.

overall (Fig 1, right panel, and Table 3). 104 (87\%) patients reported having their appointment on time (Q8), while 9 (8\%) reported delays. 117 (98\%) patients reported that the telephone consultation saved them time from travelling to the hospital (Q9). $95(80 \%)$ patients found the telephone consultation to be an acceptable way of receiving healthcare, while 8 (7\%) disagreed, and $16(13 \%)$ did not specify a preference of one over the other (Q10). 92 (77\%) patients reported that they would like to use telephone consultation after the COVID-19 outbreak; however, $13(11 \%)$ would prefer to have face-to-face appointments and $14(12 \%)$ did not express a specific preference (Q11). 114 (96\%) patients agreed that, overall, they were satisfied with their telephone consultation.

Logistic regression analysis (supplementary material, S1) showed that patients in the post radical prostatectomy clinic gave a significantly greater number of 'Excellent' or 'Agree' responses to questions $7,8,10$, and 11 relative to responses from patients in the general clinic $(p<0.05)$. A similar behaviour was observed for patients in the PSA surveillance clinic for questions 7, 10 and 11. These questions related to their personal comfort using the telephone, whether the appointment occurred at the scheduled time, whether they found telephone consultations an acceptable way to receive care, and whether they would like to continue to use telephone appointments once the COVID-19 restrictions are eased, respectively. Age was found to have a significant effect on response to question 9 , with older patients more often in agreement that telephone clinics saved them time travelling to the hospital $(P=0.02)$. No significant difference was found between ages for any other item. Gender showed no significant association to any item response in the relevant (functional or general) clinics.

\section{Discussion}

The first reported use of telemedicine can be traced back to the early twentieth century, with transmission of electrocardiograms via telephone wires. ${ }^{7}$ Since then, telemedicine has continued to evolve and has been incorporated into many specialties, including urology. ${ }^{8,9}$ In the coronavirus era, telemedicine has proven a useful tool to contain the spread of the virus. In the USA, more than 50 health institutions are using telemedicine, aiming to create a national 'forward triage' to control the surge of emergency admissions during the COVID pandemic. ${ }^{10}$

Due to the unprecedented circumstances during the COVID-19 pandemic, it was a matter of public safety to switch from face-toface appointments to telephone appointments in a 'big bang' roll out without any prior pilot studies. Recently, Luciani et al described how an Italian urology department radically reshaped their clinic list during the COVID-19 pandemic. ${ }^{5}$ They achieved this firstly by cancelling certain consultations without rescheduling them, and secondly by screening patient records and then offering either a face-to-face appointment or a remote consultation, dependent on clinical need. In contrast, our practice at the Stokes Centre for Urology was to automatically convert all outpatient face-to-face clinic appointments to telephone consultations. This afforded the evaluation of patient satisfaction amongst a wide range of different urology patients.

We found that although overall satisfaction with telephone consultations was high amongst our cohort of patients, a degree of variance was evident between some subgroups. Many patients followed up for prostate disease (either benign or malignant) expressed their preference for telephone appointments over face-to-face consultations. PSA level and imaging results can often be communicated remotely, with the advantage of convenience for the patient. This finding is consistent with a recent study that found that remote video visits for post prostatectomy patients were associated with equivalent efficiency and similar satisfaction to traditional clinic appointments, and with significantly lower patient costs. ${ }^{11}$

On analysis of age groups, we observed an association between more advanced age and greater satisfaction, specifically in relation to saving travel time. In addition, younger patients (30-50 years) appeared to exhibit lower levels of satisfaction on each of the 12 items, although this was not significant. Despite this, the distribution of patient ages among clinic type must be considered, given that the vast majority of patients who were reviewed for prostate conditions lie within the older age range, and our sample size of patients under 50 years was only eight. Hence, it is difficult to confidently rely on this 
Table 2. Responses to the adapted Telehealth Satisfaction Scale (TeSS) by type of clinic $(n=119)$

Item Statement: Clinic

How would you rate:

1 The voice quality of the appointment

2 The duration of the appointment

The explanation of the treatment

The explanation of the diagnosis

The thoroughness and carefulness of the clinician

Your personal comfort using the telephone for an appointment

\author{
Clinic
}

$$
\text { Prosta }
$$

General

Functional

Andrology

Total

Prostatectomy

PSA surveillance

General

Functional

Andrology

Total

Prostatectomy

PSA surveillance

General

Functional

Andrology

Total

Prostatectomy

PSA surveillance

General

Functional

Andrology

Total

Prostatectomy

PSA surveillance

General

Functional

Andrology

Total

Prostatectomy

PSA surveillance

General

Functional

Andrology

Total

Prostatectomy

PSA surveillance

General

Functional

Andrology

Total
Response $\mathbf{n}(\%)$

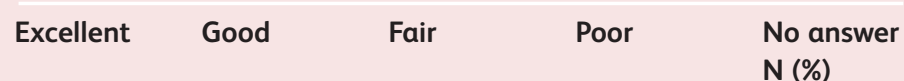

$32(89) \quad 4(11)$

$30(88)$

3 (9)

$5(21)$

$15(83)$

3 (17)

$6(86)$

$1(14)$

$102(86)$

16 (13)

29 (81)

7 (19)

$30(88)$

3 (9)

18 (75)

5 (21)

$14(78)$

3 (17)

6 (86)

0

97 (82)

18 (15)

27 (75)

1 (3)

$24(71)$

3 (9)

$16(67)$

2 (8)

$11(61)$

4 (22)

4 (57)

1 (14)

82 (69)

11 (9)

$22(61)$

1 (3)

22 (65)

3 (9)

$16(67)$

3 (13)

15 (83)

0

4 (57)

1 (14)

79 (66)

8 (7)

31 (86)

5 (14)

29 (85)

$4(12)$

16 (67)

4 (17)

15 (83)

2 (11)

6 (86)

0

97 (82)

15 (13)

$36(100)$

0

32 (94)

1 (3)

21 (88)

3 (13)

17 (94)

1 (6)

6 (86)

1 (14)

112 (94)

6 (5)

33 (92)

3 (83)

32 (94)

2 (6)

15 (83)

6 (33)

$13(72)$

3 (17)

4 (57)

2 (29)

97 (82)
0

0

0

0

0

0

0

0

1 (4)

1 (6)

1 (14)

3 (3)

1 (3)

0

1 (4)

$1(6)$

1 (14)

4 (3)

0

2 (6)

0

$1(6)$

1 (14)

4 (3)

0

0

1 (4)

1 (6)

1 (14)

3 (3)

0

1 (3)

0

0

0

1 (1)

0

0

3 (13)

2 (11)

0

5 (4)
N (\%)

$0 \quad 0$

1 (3) 0

$0 \quad 0$

$0 \quad 0$

$0 \quad 0$

1 (1) 0

$0 \quad 0$

1 (3) 0

$0 \quad 0$

$0 \quad 0$

$0 \quad 0$

1 (1) 0

$0 \quad 7$

2 (6) 5

$1(4) \quad 4$

02

0

3 (2) $\quad 19$ (16)

$0 \quad 13$

1 (3) 6

1 (4) 4

02

01

2 (2) 26 (22)

$0 \quad 0$

1 (3) 0

2 (8) 1

$0 \quad 0$

$0 \quad 0$

$\begin{array}{ll}3(3) & 1(1) \\ 0 & 0\end{array}$

$\begin{array}{ll}0 & 0 \\ 0 & 0\end{array}$

$\begin{array}{ll}0 & 0 \\ 0 & 0\end{array}$

$0 \quad 0$

$0 \quad 0$

$\begin{array}{ll}0 & 0 \\ 0 & 0\end{array}$

$\begin{array}{ll}0 & 0 \\ 0 & 0\end{array}$

0

$\begin{array}{ll}0 & 0 \\ 0 & 0\end{array}$

$\begin{array}{ll}0 & 0 \\ 0 & 0\end{array}$

0

$0 \quad 1$ (1)


Table 3. Responses to additional questions on telephone appointments by type of clinic $(n=119)$

Item Statement

8 Telephone consultation
happened on time as
scheduled

Telephone consultation saved time travelling to the hospital

10

Telephone consultations are an acceptable way to receive care

I would like to use telephone consultations after COVID-19

12

Overall, I am satisfied with my telephone consultation

Clinic

Response $\mathrm{n}(\%)$

\begin{tabular}{|c|c|c|c|c|}
\hline & Agree & $\begin{array}{l}\text { Neither agree } \\
\text { nor disagree }\end{array}$ & Disagree & No answer \\
\hline Prostatectomy & $35(97)$ & 0 & $1(3)$ & 0 \\
\hline PSA surveillance & $33(97)$ & 0 & $1(3)$ & 0 \\
\hline General & $19(79)$ & $3(13)$ & $2(8)$ & 0 \\
\hline Functional & $10(56)$ & $3(17)$ & $5(28)$ & 0 \\
\hline Andrology & $7(100)$ & 0 & 0 & 0 \\
\hline Total & $104(87)$ & $6(5)$ & $9(8)$ & 0 \\
\hline Prostatectomy & $36(100)$ & 0 & 0 & 0 \\
\hline PSA surveillance & $34(100)$ & 0 & 0 & 0 \\
\hline General & $24(100)$ & 0 & 0 & 0 \\
\hline Functional & $17(94)$ & 0 & $1(6)$ & 0 \\
\hline Andrology & $6(86)$ & $1(4)$ & 0 & 0 \\
\hline Total & $117(98)$ & $1(1)$ & $1(1)$ & 0 \\
\hline Prostatectomy & $33(92)$ & $3(8)$ & 0 & 0 \\
\hline PSA surveillance & $31(91)$ & $2(6)$ & $1(3)$ & 0 \\
\hline General & $14(58)$ & $5(21)$ & $5(21)$ & 0 \\
\hline Functional & $11(61)$ & $5(28)$ & $2(11)$ & 0 \\
\hline Andrology & $6(86)$ & $1(14)$ & 0 & 0 \\
\hline Total & $95(80)$ & $16(13)$ & $8(7)$ & 0 \\
\hline Prostatectomy & $32(89)$ & $4(11)$ & 0 & 0 \\
\hline PSA surveillance & $29(85)$ & $2(6)$ & $3(9)$ & 0 \\
\hline General & $15(63)$ & $3(13)$ & $6(25)$ & 0 \\
\hline Functional & $12(67)$ & $4(22)$ & $2(11)$ & 0 \\
\hline Andrology & $4(57)$ & $1(14)$ & $2(29)$ & 0 \\
\hline Total & $92(77)$ & $14(12)$ & $13(11)$ & 0 \\
\hline Prostatectomy & $36(100)$ & 0 & 0 & 0 \\
\hline PSA surveillance & $33(97)$ & 0 & $1(3)$ & 0 \\
\hline General & $21(88)$ & $1(4)$ & $2(8)$ & 0 \\
\hline Functional & $17(94)$ & 0 & $1(6)$ & 0 \\
\hline Andrology & $7(100)$ & 0 & 0 & 0 \\
\hline Total & $114(96)$ & $1(1)$ & $4(3)$ & 0 \\
\hline
\end{tabular}

finding. Larger studies which can allow for this compounding factor would be useful, especially if ongoing circumstances require remote consultations for our whole range of patients.

One of the limitations of our approach is the sole use of audio consultation. Video consultations may better simulate a real time experience and might increase clinician and patient trust. Video conference may also facilitate the easier identification of unwell patients. Many patients in our study reported that the use of video would improve the quality of the consultation.

We employed an adaption of a validated telehealth satisfaction questionnaire which was appropriate for our patients overall; however, the questionnaire was not piloted by our team in 
advance. Due to the heterogeneity of the cohort, questions 3 and 4 , relating to the explanation of diagnosis and treatment, were not relevant for every patient. Validated instruments for urology patients and specific patient populations will be of value in future studies, as telemedicine becomes more widespread within the specialty. However, by including additional questions we strived to gain an accurate interpretation of our patients' perspective.

Additional limitations apply to the mode of administration of the questionnaire. Responses were collected exclusively via telephone, and not in written format, which may have influenced patient responses. Telephone surveys are more often associated with both 'yes-saying' bias and interviewer bias, which may elevate reported satisfaction.12 Additionally, they may lead to an unwillingness to disclose sensitive information to the interviewer, in comparison to written questionnaires.12 Nevertheless, in view of the challenging time constraints and increased safety measures surrounding the COVID-19 pandemic, administration of written questionnaires was not feasible.

Overall, $95 \%$ of patients stated that they were satisfied with their telephone consultation, suggesting a role for telephone consultations in urology. However, a lesser $77.3 \%$ of patients reported that they would like to continue attending telephone clinics after the easing of lockdown restrictions. Clearly, telemedicine cannot completely replace face-to-face consultations, which are superior for breaking bad news and assessing acutely unwell patients. However, for long-term follow up and review of chronic conditions it is more suited, which is supported by our findings. Ultimately, many patients will opt for face-to-face consultations over telephone consultations due to personal preference, and this should be respected, with consideration given to patient and clinician safety. Thus, we believe that the option of telephone and remote consultations should be at least offered to appropriate patients as part of a patient-centred approach.

\section{Conclusion}

Our study demonstrates high overall satisfaction with the use of telephone consultations for urology patients during the COVID-19 pandemic. However, significantly greater satisfaction was measured for patients within the post radical prostatectomy and PSA surveillance clinics. This supports their continued use, to some extent, beyond the COVID-19 pandemic. However, it is clear that in selected cases face-to-face consultations are required for safe, comprehensive clinical assessment.

\section{Supplementary material}

Additional supplementary material may be found in the online version of this article at www.rcpjournals.org/fhj:

S1 - Univariable logistic regression

\section{References}

1 Simonato A, Giannarini G, Abrate A et al. Pathways for urology patients during the COVID-19 pandemic. Minerva Urol Nefrol 2020;72:376-83.

2 Ohannessian R, Duong TA, Odone A. Global telemedicine implementation and integration within health systems to fight the COVID-19 pandemic: a call to action. JMIR Public Health Surveill 2020;6:e18810.

3 World Health Organization. A health telematics policy in support of WHO's Health-For-All strategy for global health development: report of the WHO group consultation on health telematics, 11-16 December, Geneva, 1997. WHO, 1998. Available from https://apps. who.int/iris/handle/10665/63857.

$4 \mathrm{Ho} \mathrm{HC}$, Hughes T, Bozlu M et al. What do urologists need to know: Diagnosis, treatment, and follow-up during COVID-19 pandemic. Turk J Urol 2020;46:169-177.

5 Luciani LG, Mattevi D, Cai T et al. Teleurology in the Time of Covid-19 pandemic: here to stay? Urology 2020;140:4-6.

6 Morgan DG, Kosteniuk J, Stewart N et al. The Telehealth Satisfaction Scale (TeSS): Reliability, validity, and satisfaction with telehealth in a rural memory clinic population. Telemed J E Health 2014:20:997-1003.

7 Einthoven W. Le télécardiogramme [The telecardiogram]. Arch Int Physiol 1906;4:132-64.

8 Lurie N, Carr BG. The role of telehealth in the medical response to disasters. JAMA Intern Med 2018;178:745-6.

9 Safir IJ, Gabale S, David SA et al. Implementation of a tele-urology program for outpatient hematuria referrals: initial results and patient satisfaction. Urology 2016;97:33-9.

10 Hollander JE, Carr BG. Virtually perfect? Telemedicine for Covid. N Engl J Med 2020;382:1679-81.

11 Viers BR, Lightner DJ, Rivera ME f. Efficiency, satisfaction, and costs for remote video visits following radical prostatectomy: a randomized controlled trial. Eur Urol 2015;68:729-35.

12 Bowling A. Mode of questionnaire administration can have serious effects on data quality. J Public Health (Oxf) 2005;27:281-91.

Address for correspondence: Dr Tharani Mahesan, Stokes Centre for Urology, Royal Surrey County Hospital, Egerton Road, Guildford, Surrey GU2 7XX, UK.

Email: tharani.mahesan@nhs.net 